21. The ordinary discriminant of the ternary quadratic form $a_{. x}^{2}+b_{x} x_{3}+c x_{3}^{2}$ is expressible as the resultant of the binary forms $a_{x}^{2}, b_{x}$ plus $c$ times the discriminant of $a_{x}^{2}$. Professor Glenn proves the corresponding general theorem for the ternary form of order $m$, and derives explicit formulas for the $\frac{1}{2} m$ $(m-1)$ ordinary discriminants (conditions that the forms degenerate into distinct linear factors) in terms of the coefficients of the form. One of these discriminants is a linear expression in the resultant of $a_{x}^{m}$ and $b_{x}^{m-1}$ and the discriminant of $a_{x}^{m}$, while the rest are obtained from this one by operating by powers of

and

$$
\Delta_{1}=m a_{0} \frac{\partial}{\partial b_{0}}+(m-1) a_{1} \frac{\partial}{\partial \bar{b}_{1}}+\cdots+a_{m-1} \frac{\partial}{\partial b_{m-1}}
$$

$$
\Delta_{2}=m a_{m} \frac{\partial}{\partial b_{m-1}}+(m-1) a_{m-1} \frac{\partial}{\partial b_{m-2}}+\cdots+a_{1} \frac{\partial}{\partial b_{0}} .
$$

He also considers the explicit form of the satellite form of the $m$-ic and its character as an invariant.

$$
\begin{aligned}
& \text { F. N. Cole, } \\
& \text { Secretary. }
\end{aligned}
$$

\title{
THE WINTER MEETING OF THE CHICAGO SECTION.
}

The twenty-eighth regular meeting of the Chicago Section of the American Mathematical Society was held at the University of Minnesota, Minneapolis, Minn., on Wednesday, Thursday, and Friday, December 28-30, 1910, in affiliation with the American Association for the Advancement of Science. Five half-day sessions were held, beginning Wednesday afternoon with a meeting in connection with Section A (mathematics and astronomy) to hear the address of the retiring vicepresident, Professor E. W. Brown of Yale University, on "The relations of Jupiter with the asteroids." Other papers of a mathematical character at this session and at its continuation on Thursday morning were by Professor F. R. Moulton of the University by Chicago on "The debt of mathematics to astronomy," and of Dr. W. D. Macmillan of the University of Chicago on "An integrable case in the problem of three bodies." The vice-president of Section A, Professor E. H. Moore of the University of Chicago, presided throughout this session. 
The last half-day session, on Friday afternoon, was also a joint meeting with Section A and with Sections B (physics) and $\mathrm{D}$ (mechanical engineering) of the American Association, to hear the report of Professor E. V. Huntington of Harvard University, chairman of the committee of twenty appointed at a similar meeting three years ago at Chicago, on "The teaching of mathematics to students of engineering." The work of this committee has extended along two distinct lines, first, the gathering of statistics on this subject from about forty schools where engineering is taught in this country, and secondly, the compilation of a set of syllabi on algebra, geometry, trigonometry, dynamics, and numerical computation. The statistics were turned over to a similar committee working under the International Commission on the teaching of mathematics, and the syllabi formed the basis of Professor Huntington's report at this meeting. They have been printed in preliminary form through the cooperation of the Society for the Promotion of Engineering Education and were presented to all members of this conference for criticisms and suggestions either at the meeting or at any time later to the chairman; for this purpose additional copies may be had on request from the chairman. About forty engineers, physicists and mathematicians attended this meeting and a most interesting and animated discussion was participated in by a large number including Professors E. J. Wilczynski, University of Chicago; A. G. Hall, University of Michigan ; E. H. Comstock, School of Mines, University of Minnesota; G. A. Miller, University of Illinois; A. E. Haynes, College of Engineering, University of Minnesota; E. F. Nichols, president of Dartmouth College; T. F. Holgate, Northwestern University; Alexander Ziwet, University of Michigan ; E. R. Maurer, College of Engineering, University of Wisconsin ; Henry Crew, Northwestern University; $H$. E. Slaught, University of Chicago ; B. L. Newkirk, University of Minnesota; E. F. Chandler, University of North Dakota; J. J. Flather, head of the department of mechanical engineering, University of Minnesota, and I. E. Dickson, University of Chicago, who presided at the meeting. Also numerous criticisms of the report were presented in writing either directly to the chairman or through the secretary of the meeting. Among these were communications from President R. S. Woodward of Washington, and Professor William Kent of New York. In conclusion it was resolved that the members 
of this body tender hearty thanks to Professor Huntington for the great interest which he has shown in this work and the untiring service which he has given to it, that they commend the work of the whole committee for the preparation of a report which it is believed must operate for betterment along the lines of the recommendations, and while not prepared to approve in all respects the details, especially in the syllabus on dynamics, as shown by the full and free discussion at this meeting, yet they heartily endorse the spirit of the report and thank the officers of the Society for the Promotion of Engineering Education who have shown their friendly cooperation in offering to publish these syllabi in their official Bulletin for the purpose of drawing out further criticisms and suggestions either in printed papers or in written communications to the chairman of the committee. The further report of this committee is to be presented at the next meeting of the Society for the Promotion of Engineering Education in June, 1911.

The remaining three sessions of the Chicago Section were devoted to the reading of mathematical papers. Professor L. E. Dickson, chairman of the section, presided except when relieved, during the reading of his own papers, by Professor Alexander Ziwet and by Professor A. G. Hall. The total attendance of forty-five at these sessions included the following thirty members of the society:

Professor G. N. Bauer, Professor W. E. Brooke, Professor E. W. Brown, Professor G. L. Brown, Professor W. H. Bussey, Professor E. H. Comstock, Professor H. H. Dalaker, Professor L. E. Dickson, Professor J. F. Downey, Professor H. T. Eddy, Professor Peter Field, Professor A. G. Hall, Professor G. W. Hartwell, Professor T. F. Holgate, Professor E. V. Huntington, Professor Kurt Laves, President E. O. Lovett, Professor G. A. Miller, Dr. W. D. MacMillan, Professor E. H. Moore, Professor F. R. Moulton, Dr. L. I. Neikirk, Professor B. L. Newkirk, Professor W. J. Risley, Professor R. R. Shumway, Professor H. E. Slaught, Dr. H. L. Slobin, Dr. E. H. Taylor, Professor E. J. Wilczynski, Professor Alexander Ziwet.

On Thursday evening about one hundred of the astronomers, engineers, physicists, and mathematicians dined together at the Commercial Club and fulfilled in every respect the desire, expressed at the April meeting of the Chicago Section, for a closer affiliation between these representatives of the American 
Association. Professor E. H. Moore acted as toastmaster and impromptu addresses were made by Dean F. C. Shenehon, of the School of Engineering, University of Minnesota, and President C. S. Howe, of Case School of Applied Science, representing the engineers; by President E. F. Nichols, of Dartmouth College, and Professor E. L. Nichols, of Cornell University, representing the physicists; and by Professor E. V. Huntington, of Harvard University, and Professor E. W. Brown, of Yale University, representing the mathematicians.

The election of officers of the Section for the ensuing year resulted as follows: chairman, Professor L. E. Dickson, secretary, Professor H. E. Slaught, third member of the program committee Professor J. B. Shaw. It was voted that the chairman of the Section be requested to deliver an address on the occasion of retiring from his office at the winter meeting in 1911.

The following papers were read at this meeting:

(1) Professor G. A. Miriser: "On the use of the co-sets of a group."

(2) Professor I. E. Dickson : "Congruencial theory of functions of several variables."

(3) Professor L. E. Dickson : "Generalization of theorems on linear algebras."

(4) Professor JАCOB Westlund : "On primitive roots of ideals."

(5) Dr. A. B. Frizels : "The problem of defining the set of real numbers."

(6) Professor Florian Cajori : "A historical note on the Newton-Raphson method of approximations."

(7) Professor E. J. Wriczynski : "One-parameter families and nets of plane curves."

(8) Dr. H.T. Burgess : "Circular numbers for a plane curve."

(9) Dr. H. T Buraess: "Rational anharmonic curves upon a quadric."

(10) Dr. H. T. Burgess: "The applications of matrices to cubic forms."

(11) Professor W. J. Risley and Professor W. E. MacDonaLD : "Envelopes of one-parameter families of plane curves."

(12) Dr. W. D. MacMillan : "A reduction of two power series in many variables to two equivalent polynomials."

(13) Dr. H. F. MACNEISH : "The path of light in a medium homogeneous in concentric spherical layers." 
292 Winter meeting of the chicago section. [March,

(14) Professor Kurt Laves: "The curves of equal action for elliptical coordinates."

(15) Professor L. C. Karpinski : "Robert of Chester's translations of the algebra of Al-Khowarazmi."

(16) Professor L. C. KaRPinski: "Hindu numerals in the Kitab al Fihrist."

(17) Dr. Arthur Ranum: "Ruled surfaces and planed hypersurfaces in four dimensional space."

(18) Dr. L. I. NeIkiRK : "Transformation groups and substitutions of an infinite degree."

(19) Professor J. B. Shaw : "Use of quaternions in differential geometry."

(20) Dr. H. L. Slobin : "On plane quintic curves."

(21) Professor F. R. Moulton: "On the construction of a certain class of periodic solutions of the problem of three bodies."

(22) Professor F. B. Williams: "Curves on quintic scrolls."

Professor Williams was introduced by Professor G. A. Miller. In the absence of the authors the papers of Professors Cajori, Westlund, Karpinski, Shaw, and Williams, Drs. Frizell, Burgess, MacNeish, and Ranum were read by title. Abstracts of the papers follow below, the numbers corresponding to those in the above list.

1. If $H$ is any subgroup of the group $G$, the operators $S_{2}, S_{3}, \ldots, S_{\rho}$ may be so selected that every operator of $G$ is found once and only once in each of the following two sets :

$$
\begin{aligned}
& H+H S_{2}+H S_{3}+\cdots+H S_{\rho}, \\
& H+S_{2} H+S_{3} H+\cdots+S_{\rho} H .
\end{aligned}
$$

The sets $H S_{a}, S_{a} H(\alpha=2,3, \cdots, \rho)$ are called the co-sets of $G$ as regards $H$, the former being called right co-sets and the latter left co-sets. When $H$ is added to these co-sets they are called respectively right and left augmented co-sets. The operators of $G$ may also be represented uniquely in each of the following two ways, $H_{1}$ and $H_{2}$ being two arbitrary subgroups of $G$ :

$$
\begin{aligned}
& H_{1} H_{2}+H_{1} S_{2} H_{2}+\cdots+H_{1} S_{\lambda} H_{2}, \\
& H_{2} H_{1}+H_{2} S_{2}^{-1} H_{1}+\cdots+H_{2} S_{\lambda}^{-1} H_{1} .
\end{aligned}
$$

The sets $H_{1} S_{a} H_{2}(\alpha=2,3, \ldots, \lambda)$ are called double co-sets of $G$ as regards $H_{1}$ and $H_{2}$, and when $H_{1} H_{2}$ is added they are called the augmented double co-sets as regards these subgroups. 
The objects of Professor Miller's paper are to exhibit how a number of known theorems may readily be proved and related by employing these concepts explicitly, and especially to derive some important relations between abstract groups and substitution groups. The following theorems are proved : (1) If $G$ is represented as a transitive substitution group $K$, and if $K_{1}$ is the subgroup composed of all the substitutions of $K$ which omit a given letter, then any subgroup $K^{\prime}$ of $K$ has the same number of transitive constituents as the subgroup which corresponds to $K_{1}$ has when $K$ is represented with respect to $K^{\prime}$. Moreover, the transitive constituents in the two given subgroups have the same relative degrees in the two given representations of $G$. (2) The product $H_{1} H_{2}$ cannot involve the inverse of each operator in a double co-set with respect to $H_{1}, H_{2}$.

2. The term function is used, for brevity, in Professor Dickson's first paper to designate a rational integral function with integral coefficients. If $p$ is a prime, two functions $f$ and $g$ of a single variable $x$ have, modulo $p$, a greatest common divisor $d(x)$ which is unique apart from a constant factor and may be determined by an obvious modification of Euclid's algorithm. We have only to multiply the divisor by such a constant that the new leading coefficient is $\equiv 1(\bmod p)$. The same method does not apply for functions of two or more variables, nor are the resulting theorems entirely analogous to those holding for a single variable. In the latter case $d(x)$ is a linear combination of $f$ and $g$ modulo $p$. But for $f=(x+1) y-1$, $g=x y+x$ and $p=3, x f-(x+1) g=-x(x+2)$, so that the greatest common divisor of $f$ and $g$ is a constant prime to 3 ; and we do not have $1 \equiv A f+B g(\bmod 3)$, since for $x \equiv 1$, $y \equiv-1$, we have $f \equiv g \equiv 0(\bmod 3)$.

To construct a congruencial theory of functions of two variables (and then by induction for $n$ variables), denote by $d(x)$ the greatest common divisor modulo $p$ of the coefficients $f_{i}(x)$ in $f=\Sigma f_{i}(x) y^{i}$ and call $f$ primitive in $y$ modulo $p$ when $d(x)$ is a constant not divisible by $p$. We readily show that if $f g$ is divisible modulo $p$ by a function $P(x)$, irreducible modulo $p$, either $f$ or $g$ is divisible modulo $p$ by $P(x)$. It follows that the product of two functions primitive in $y$ modulo $p$ is primitive in $y$. If $\phi(x) F$ is divisible modulo $p$ by $G$, where $F$ and $G$ are primitive in $y$, then $F$ is divisible by $G$. A function primitive in $y$ modulo $p$ has as divisors only functions primitive in $y$. 
To establish the existence of a greatest common divisor $(f, g)$ modulo $p$ of $f$ and $g$, let $g \equiv N(\bmod p), N^{\prime}=V y^{n}+V^{\prime} y^{n-1}+\cdots$, where $V, V^{\prime}, \ldots$ are functions of $x$ only, and $V \neq 0(\bmod p)$. For a suitable integer $k$, we have $V^{k} f=N q_{1}+r_{1}$, algebraically, where $r_{1}$ is of degree $<n$ in $y$. In this way we get

$$
V^{k} f \equiv g q_{1}+r_{1}, \quad V_{1}^{k_{1}} g \equiv r_{1} q_{2}+r_{2}, \cdots(\bmod p) .
$$

Let $r_{m}$ be the last remainder not divisible by $p$. Let $f=\phi_{(m)}^{m} F, g=\gamma(x) G, r_{m}=\rho(x) R$, where $F, G, R$ are primitive in $y$ modulo $p$. By use of the preceding lemmas, we readily prove that $R=(F, G),(f, g)=G(\phi, \gamma)$. We may now establish the unique factorization theorem.

The same method is applicable to the theory of algebraic forms ; in particular, it avoids the use of Kronecker's theorem concerning the coefficients of the product of two polynomials.

3. The second paper by Professor Dickson deals with linear algebras in which multiplication is not assumed to be associative or commutative. Given two numbers $X$ and $Y$ of the algebra, $X \neq 0$, we can determine a number $X^{\prime}$ such that $X X^{\prime}=Y$ or $X^{\prime} X=Y$ if and only if the right hand or left hand determinant

$$
\Delta(X)=\left|\sum_{i=1}^{n} x_{i} \gamma_{i j k}\right|, \quad \Delta^{\prime}(X)=\left|\sum_{i=1}^{n} x_{i} \gamma_{j i l_{k}}\right| \quad .
$$

is not zero. Here the $\gamma_{i j / k}$ are the constants of multiplication. We assume that the unit $e_{1}$ is a modulus. Then $x_{1}$ occurs only in the terms of the main diagonal of $\Delta$ or $\Delta^{\prime}$. Replacing $x_{1}$ by $x_{1}-\rho$, we obtain the right and left hand characteristic determinants of $X$

$$
\Delta(X-\rho)=\sum_{i=0}^{n} r_{i} \rho^{i}, \quad \Delta^{\prime}(X-\rho)=\sum_{i=0}^{n} l_{i} \rho^{i} .
$$

Set $X_{1}=X, X_{2}=X^{2}, X_{3}=X_{2} X, X_{i+1}=X_{i} X$. Similarly, set ${ }_{i+1} X=X\left({ }_{i} X\right)$. Take $x_{1}^{\prime}, \ldots, x_{n}^{\prime}$ to be the co-factors of the elements in the first row of $\Delta^{\prime}(X)^{n}$. Multiply them by the respective elements of the $k$-th row of $\Delta^{\prime}(X)$ and add. We obtain the coefficient $y_{l_{0}}$ of $Y=X^{\prime} X$. Hence $y_{1}=\Delta^{\prime}, y_{k}=0(k>1)$. Thus $X^{\prime} X=\Delta^{\prime}$. Replace $x_{1}$ by $x_{1}-\rho$ and let $X^{\prime \prime}$ become

$$
F=\sum_{i=0}^{n-1} F_{i}^{\prime} \rho^{i}
$$


where $F_{i}$ are numbers of the algebra. Thus

$$
F(X-\rho)=\sum_{i=0}^{n} l_{i} \rho^{i}
$$

By equating coefficients of like powers of $\rho$, we get

$$
\begin{aligned}
F_{0} X=l_{1}, F_{1} X-F_{0}=l_{1}, F_{2} X-F_{1}=l_{2}, \cdots, \\
F_{n-1} X-F_{n-2}=l_{n-1},-F_{n-1}=l_{n} .
\end{aligned}
$$

Multiply the second on the right by $X$, the third by $X$ twice, the fourth by $X$ three times, etc. Adding we get

$$
\sum_{i=0}^{n} l_{i} X_{i}=0
$$

Hence the left-hand characteristic determinant of $X$ vanishes if we replace $\rho^{i}$ by $X_{i}$ (i. e., it has $X$ as a right-hand root). Similarly, any element $X$ is a left-hand root of the right-hand characteristic determinant.

Various properties of polynomials in $\rho$ have their analogue in those of the linear combinations of the right hand powers $\boldsymbol{X}_{\boldsymbol{i}}$ (or the left hand powers of $X$ ). If

we set

$$
A_{0}=\sum_{i=0}^{a} a_{i} X_{i}
$$

$$
A_{k}=\sum_{i=0}^{\alpha} a_{i} X_{i+l k}
$$

whence $A_{k}=A_{l-1} X$. Then if $\Sigma a_{i} \rho^{i}$ and $\Sigma b_{i} \rho^{i}$ have the greatest common divisor $\Sigma g_{i} \rho^{i}$, we have $A_{0}=\Sigma m_{s} G_{s}, B_{0}=\Sigma n_{t} G_{t}$, while $G_{0}=\Sigma p_{s} A_{s}+\Sigma q_{t} B_{t}$, in which the $m, n, p, q$ are scalars, $B_{0}=\Sigma b_{i} X_{i}^{s}, \quad G_{0}=\Sigma g_{i} X_{i}$. In particular, $A_{0}$ and $B_{0}$ both vanish if and only if $G_{0}$ vanishes. Various other results follow in the usual manner.

4. Every prime ideal $P$ in an arbitrary algebraic number field admits, as is well known, of primitive roots. But for $P^{n}$, where $n>1$, primitive roots do not always exist. In what cases primitive roots of $P^{n}$ exist was determined by $A$. Wiman in 1899.* It is the object of Professor Westlund's paper to treat the somewhat more general problem of actually determining the exponent, $\bmod P^{n}$, to which any primitive root of $P$ belongs.

\footnotetext{
* Öfversigt af kongliga vetenskapsakademiens Förhandlingar, vol. 56.
} 
5. Studies in the continuum perhaps too often ignore Hilbert's pregnant remark that the totality of real numbers has not yet been defined. Any scheme for producing transfinite series of fundamental sequences is nothing more nor less than a process of defining new sets of irrational numbers. It is a problem of epistemology whether the human intellect can ever exhaust the possibilities of such definition. Dr. Frizell considers the following example.

I et us define inductively a function of $2,3, \ldots, n$ arguments

$$
e(\alpha, \beta)=\alpha^{\beta}, \quad e\left(\alpha_{i+1}, \alpha_{i}, \cdots, \alpha_{1}\right)=e\left\{\alpha_{i+1}, e\left(\alpha_{i}, \cdots, \alpha_{1}\right)\right\}
$$

for example,

$$
(i=1,2, \cdots, n-1) \text {, }
$$

$$
e(2,3,5)=2^{243} \text {. }
$$

First let the values of $x_{i}$ be the well ordered set of positive fractional numbers (integers included, except unity). Next let each $\alpha_{i}$ take in succession all the above values of the $e$ function and repeat the procedure indefinitely.

Now define a new function $f$ such that for integral values of $n$ $f(\alpha, n)=e\left(\alpha, \alpha, \ldots, n\right.$ arguments), e. g., $f(2,5)=2^{65536}$. If we succeed in defining $f(\alpha, \beta)$ for all values of $\alpha, \beta$ in the set defined above by the $e$ function, there will follow the problem of defining in like manner a function $f_{1}$ so that $f_{1}(\alpha, n)=$ $f(\alpha, \alpha, \cdots,(n$ arguments $))$, and so on. But we cannot define all such functions.

6. In Professor Cajori's paper it is shown that what, since the time of Fourier, has been called "Newton's method" of approximation to the roots of numerical equations is not Newton's method, but Raphson's modification of it. Before 1800 the methods of Newton and Raphson were almost invariably kept distinct. Professor Cajori suggests that the approximation, as described in modern texts, be called the "Newton-Raphson method."

This paper will appear in full in the February number of the American Mathematical Monthly.

7. In most treatments of one-parameter families and nets of plane curves, the interest is concentrated altogether upon the determination and discussion of its envelope. More recently some authors, especially Lilienthal and Scheffers, have discussed a number of other interesting problems associated with such 
families of curves, but all of these are of a metric nature. In Professor Wilczynski's paper the point of view of projective differential geometry has been adopted, the analytical treatment being based upon the invariant theory of a completely integrable system of partial differential equations of the form

$$
\begin{aligned}
& y_{u u}=a y_{u}+b y_{v}+c y, \\
& y_{u v}=a^{\prime} y_{u}+b^{\prime} y_{v}+c^{\prime} y, \\
& y_{v v}=a^{\prime \prime} y_{u}+b^{\prime \prime} y_{v}+c^{\prime \prime} y .
\end{aligned}
$$

The second equation of this system is what Darboux calls a Laplacian equation, and there exists an extensive theory concerned primarily with the cases in which it may be integrated by quadratures. This theory here finds a new and simple interpretation. Moreover, the fact that this equation appears here merely as one of three, instead of by itself, adds deeply to the significance of the theory.

Other points discussed in the paper are the determination of a complete set of invariants and covariants, the osculating conics of the individual curves of the net and their mutual relations.

8. In this paper Dr. Burgess shows that the set of circular numbers defined below are arithmetical invariants of a plane curve under the transformations of the circular group $G_{6}$. The small letters are Plücker's well-known numbers.

$M$ : The degree of $C$ is the number of points of $C$ in common with an arbitrary circle. For a circular curve, $M=\mu$.

$R$ : The class of $C$ is the number of circles of an arbitrary pencil touching $C$. For a circular curve, $R=\nu$.

$N$ : The inflexion of $C$ is the number of osculating circles of $C$ through an arbitrary point. For a circular curve, $N=i$.

$A$ : The number of stationary circles of $C$. The same for a circular curve.

$B$ : The cuspidal equivalence of $C$. For a circular curve, $B=\kappa$.

$X$ : The number of doubly orthogonal circles of $C$ through an arbitrary point. For a circular curve, $X=$ the number of binormals.

$Y$ : The number of bitangent circles of $C$ through an arbitrary point. For a circular curve, $Y=\tau$.

$G$ : The number of pairs of osculating circles of $C$ which intersect upon an arbitrary circle. For a circular curve, $G$ is the number of pairs of osculating circles having a common radical axis. 
$H$ : The number of pairs of tangent circles of $C$ belonging to an arbitrary net while each circle of the pair passes through the point of contact of the other. For a circular curve all the circles may have their centers upon an arbitrary straight line.

$D$ : The nodal equivalence. For a circular curve, $D+H=\delta$. $I$ : The number of intersections of $C$ with a minimum line through $I$, excluding the point $I$.

$J$ : The number of intersections of $C$ with a minimum line through $J$, excluding the point $J$.

$F$ : The number of foci of $C, F=(R-21)(R-2 J)$.

9. In his second paper Dr. Burgess proves the following theorem: The necessary and sufficient condition that the anharmonic curves upon the quadric $Q \equiv \sum a_{i k} x_{i} x_{k}=0,\left|a_{i k}\right| \neq 0$, whose tangents belong to the null system $N \equiv \sum b_{i_{i}} x_{i} y_{k}=0$ may be rational skew curves of degree $n$, when the matrix $\left\|\lambda \alpha_{i_{k}}+b_{i_{k}}\right\|$ has the characteristic [1111], is that the two positive roots $\alpha$ and $\beta$ of the equation $\left|\lambda a_{i_{k}}+b_{i_{k}}\right|=0$ be in the ratio $(n-2): n$.

Proof: The canonical forms in the case [1111] may be taken as $Q \equiv x_{1} x_{4}+x_{2} x_{3}=0, N \equiv \alpha p_{14}+\beta p_{23}=0$; and $Q$ has the parametric equations $X_{1}: X_{2}: X_{3}: X_{4}=u v: u: v:-1$. Determining from these $\rho p_{14}=X_{1} d x_{4}-X_{4} d x_{1}=u d v+v d u$ and $\rho p_{23}=X_{2} d x_{3}-x_{3} d x_{2}=u d v-v d u$, he finds the differential equation of the curves to be $\alpha(u d v+v d u)+\beta(u d v-v d u)=0$.

On integrating, the curves are $u=c v^{\stackrel{\beta+a}{\beta-\alpha}}$, whence in the parameter $v$ the curves are given by

$$
\rho X_{1}=c v^{\left(\frac{\beta+a}{\beta-a}+1\right.}, \rho X_{2}=c v^{\frac{\beta+a}{\beta-a}}, \rho X_{3} v, \rho X_{4}=-1 .
$$

Oloviously the necessary and sufficient condition that the curves be rational and of degree $n$ is

$$
\frac{\beta+\alpha}{\beta-\alpha}+1=n, \text { whence } \frac{\alpha}{\beta}=\frac{n-2}{n} .
$$

In like manner, he finds in the other canonical forms that the cases $[(11) 11],[(11)(11],[(22)]$ and $[(31)]$ are rational and of degrees $2,1,1$, and 2 respectively. The one remaining form [22] has the curves

$$
X_{1}: X_{2}: X_{3}: X_{4}=c u e^{2 a u}: u: c e^{2 a u}:-1,
$$

and these are not rational for any value of the root $\alpha$. 
10. In his third paper, Dr. Burgess derives the following method for carrying out a linear substitution with matrix $\ddot{H}$ upon a cubic form $\sum a_{i j k} x_{i} x_{j} x_{l_{i}}=0(i, j, k=1,2, \cdots, n)$.

Consider the cubical matrix $\left\|a_{i j k}\right\|$ and build from it the series of plane matrices $A_{q} \equiv\left\|a_{i j q}\right\|(q=1,2, \cdots, n)$. Compute the series of matrix products $H^{\prime} A_{q} H(q=1,2, \ldots, n), H^{\prime}$ being the conjugate of $H$. From this series, build the new series $B_{p} \equiv\left\|b_{i p k}\right\|(p=1,2, \cdots, n)$ by writing for the $r$ th column of $B_{p}^{p}$ the $p$ th column of $H^{\prime} A_{r} H(r=1,2 \cdots, n)$. Compute from this last series the set of matrix products $B_{p} H\|\equiv\| c_{i p k}$ $(p=1,2, \cdots, n)$; and construct from these the cubical matrix $\left\|c_{i j k}\right\|$. This is the cubical matrix of the transformed cubic form, $\sum C_{i j k} x_{i}^{\prime} x_{j}^{\prime} x_{k}^{\prime}=0$.

11. Professors Risley and MacDonald discuss the conditions under which a one-parameter family of plane curves will possess an envelope. The discussion is exhaustive for the curves $y=f(x, \alpha)$, and partial for the curves $F(x, y, \alpha)=0$. The treatment is applicable whether the variables are real or complex.

The authors obtain a necessary and sufficient condition that

$$
P(x, y) \equiv y^{n}+\sum_{i=1}^{n} A_{i}(x) \cdot y^{n-1}
$$

be irreducible at $\left(x_{0}, y_{0}=0\right)$. They then use this theorem and Weierstrass's implicit function theorem to establish a theorem which summarizes and exhausts the facts concerning envelopes of the curves $y=f(x, \alpha)$.

For the curves $F(x, y, \alpha)$, the authors consider two cases: (a) that in which the curves have no multiple points in the region in question, (b) that in which the curves have these multiple points of order two only. They obtain sufficient conditions for an envelope in case $(a)$. Under case $(b)$ they obtain sufficient conditions for the existence of a nodal locus, and conditions necessary and sufficient that a locus of double points be an envelope locus.

12. Let us say that a power series $f\left(x_{1}, \cdots, x_{s} ; z_{1}, \cdots, z_{p}\right)$ is of order $m$ in $x_{1}, \cdots, x_{8}$ provided the terms of lowest degree in $f\left(x_{1}, \cdots, x_{s} ; 0, \ldots, 0\right)$ are of degree $m$ in $x_{1}, \ldots, x_{s}$.

There is a theorem of Weierstrass to the effect that a power series $f\left(x ; z_{1}, \cdots, z_{p}\right)$ of order $n$ in $x$ can be factored in the 
form $f\left(x ; z_{1}, \cdots, z_{p}\right)=\left[x^{n}+a_{1} x^{n-1}+\cdots+a_{n}\right] \cdot g\left(x ; z_{1}, \cdots, z_{p}\right)$, where $g\left(x ; z_{1}, \cdots, z_{p}\right)$ is a power series in $x ; z_{1}, \cdots, z_{p}$ with a constant term distinct from zero, and $a_{1}, \ldots, a_{n}$ are power series in $z_{1}, \cdots, z_{p}$ vanishing for $z_{1}=\cdots=z_{p}=0$. Since $g$ does not vanish for $x=z_{1}=\cdots=z_{p}=0$ it follows that for solutions in the neighborhood of the origin the equations $f=0$ and $x^{n}+a_{1} x^{n-1}+\cdots+a_{n}=0$ are equivalent.

It is the purpose of Dr. MacMillan's paper to show that if $f_{1}\left(x, y ; z_{1}, \cdots, z_{p}\right)$ and $f_{2}\left(x, y ; z_{1}, \cdots, z_{p}\right)$ are two power series of order $m$ and $n$ respectively in $x$ and $y$ and if a certain eliminant $E$ is distinct from zero, then two polynomials $p_{1}(x, y)$ and $p_{2}(x, y)$ whose coefficients are convergent power series in $z_{1}, \cdots, z_{p}$, can be found such that in the neighborhood of the origin the system of equations $p_{1}=p_{2}=0$ armits the same solutions for $x$ and $y$ as functions of $z_{1}, \cdots, z_{p}$ as does the system $f_{1}=f_{2}=0$, and therefore under the hypothesis that $E \neq 0$ the system $f_{1}=f_{2}=0$ admits $m n$ solutions for $x$ and $y$ as functions of $z_{1}, \cdots, z_{p}$ which vanish for $z_{1}=\cdots=z_{p}=0$. These results can be used to show that three power series in three dependent variables of order $l, m$, and $n$ respectively admit in general $\operatorname{lm} n$ solutions in the neighborhood of the origin.

13. Dr. MacNeish's paper considers the determination by means of the calculus of variations of the path of light in a medium homogeneous in concentric spherical layers. The question of conjugate points for the various types of paths is especially considered. When the absolute index of refraction $n$ is a continuous function of the position, the path of any ray in the medium is such as to make the integral

$$
I=\int_{s_{1}}^{s_{2}} n d s
$$

where $s$ is the length of the path, a minimum or a maximum according to the curvature of the refracting surface. If the medium is homogeneous in concentric spherical layers, the path minimizes the integral $I$. In this case the path lies in a plane through the center of the spheres. The problem is taken up in the plane of the path and six types of paths are obtained, designated types $A, B, C, D, E, F$, of which only paths of type $E$ may have conjugate points. A path of type $E$ ascends and reaches a maximum and then descends symmetrically to the level of the initial point. If the amplitude of the maximum 
point increases with the initial horizontal angle of the path, there are no conjugate points on the path. If the amplitude of the maximum point decreases as the initial horizontal angle of the path increases, the maximum point is a conjugate point for the path.

14. The paper of Professor Laves is a continuation of a former paper published by him in the Astronomische Nachrichten. Besides giving a simple geometrical interpretation of Haekel's theorem concerning the integration of Jacobi's partial differential equation for conservative systems, it takes up certain analytical results of Jacobi for elliptical coordinates and shows how they can be simplified by the same geometric conception.

15. The first systematic exposition of algebra was doubtless that written in the early part of the ninth century by Mohammed ibn Musa al-Khowārazmī. Translations from the Arabic into Latin were made in the first half of the twelfth century. One version, probably by Gerhard of Cremona, has been published by Libri in his Histoire des Sciences mathématiques en Italie. An independent version by an Englishman, Robert of Chester - probably also known as Robert of Reading - exists in manuscript in Vienna, in Dresden, and a third copy in the Columbia University library. Professor Karpinski presents a report on the content of this version, showing also that the two Latin versions are doubtless from the same Arabic original of which Frederic Rosen in 1830 published the text and English translation. While several references to the work by Robert of Chester have appeared, no other study of the content has been made.

16. The question of the origin of the Hindu-Arabic numerals is complicated by the fact that two forms of numerals with place value were used by Arabic writers of the tenth and succeeding centuries. Of these the gobār or dust forms resemble more nearly the ones which we use, while the socalled Hindu forms are more like the ones used by the modern Arabs. Sometimes, with both forms, superposed dots were employed instead of zeros to indicate multiples of the powers of ten, as 5 for 5000. Professor Karpinski has found in the Kitāb al-Fihrist an entirely new and hitherto uncited usage of subscript dots with the same force as the superposed dots. 
302 Winter MeEting of the Chicago section. [March,

The reference is of further importance as being one of the earliest (987 A.D.) discussions of the origin of the numerals.

17. Defining a ruled surface in four-dimensional space as a two-dimensional continuum made up of $\infty^{1}$ lines, and a planed hypersurface as a three-dimensional continuum made up of $\infty^{1}$ planes, Dr. Ranum derives some of the projective properties of these correlative loci. For instance, he shows that every non-developable ruled surface $R$ lies on one and only one developable planed hypersurface $P$. Given $P$, an infinite number of $R$ 's exist, arranged in pairs of conjugates such that every generator of one meets three consecutive generators of the other; any two conjugates meet in a curve whose tangent surface (developable) also lies on $P$. Dually, every non-developable planed hypersurface $H$ contains one and only one developable surface $S$. Given $S$, an infinite number of $H$ 's exist, arranged in pairs of conjugates such that every generating plane of one meets three consecutive generating planes of the other in straight lines; the envelope of the common tangent hyperplanes of any two conjugates is a developable hypersurface, which also contains $S$.

18. Dr. Neikirk's paper generalizes and extends the results published by him in the Transactions for July, 1910. It is shown in the first part that every transformation group defines a substitution group of finite or infinite degree. The problem of constructing the transformation, having given the substitution, is considered and solved in certain cases. The second part is devoted to the discussion of the properties of substitutions of an infinite degree.

19. In Professor Shaw's paper, the advantage of introducing into the analytic treatment of geometry of a surface the differential operator $\delta$, which is a two-dimensional variable Hamiltonian operator analogous to a variable $\nabla$, is shown. The operator $\delta$ is defined thus : Let $\rho=\phi\left(u_{1}, u_{2}\right)$, then $d \rho=\rho_{1} d u_{1}+\rho_{2} d u_{2}$. The normal is $\nu=V \cdot \rho_{1} \rho_{2}$. Then

$$
\delta=\frac{1}{T_{\nu}}\left(\rho_{2} \frac{\partial}{\partial u_{1}}-\rho_{1} \frac{\partial}{\partial u_{2}}\right) .
$$

If $\psi\left(u_{1}, u_{2}\right)=c$ is the equation of a curve on the surface, its tangent is $\delta \psi$. In any direction $\alpha$ tangent to the surface, the differential of $\psi$ is $d_{a} \psi=T V a \delta \psi$. Evidently $d_{a} \psi$ is a maxi- 
mum for $\alpha \perp \delta \psi$. If $\psi$ is called a "potential " function for the surface, $\psi=c$ gives the lines of equipotential and $V \nu \delta \psi$ the lines of flow.

The differential parameters $\Delta_{1}(\psi), \Delta_{1}(\psi, \theta), \Theta(\psi, \theta)$ are $\Delta_{1}(\psi)=-(\delta \psi)^{2}, \quad \Delta_{1}(\psi, \theta)=-S \delta \psi \delta \theta, \quad \Theta(\psi, \theta)=T V \delta \psi \delta \theta$. The differential parameter $\Delta_{2}(\psi)=-S \cdot \delta^{2} \cdot \psi$, where $\delta^{2} \psi=\delta(\delta \psi)$, remembering that $\delta$ is to be differentiated also. The geodetic curvature is $1 / \rho_{g}=S \cdot \delta U \delta \psi$. Around any area on the surface we have

$$
\int d \rho q=\iint^{\circ} \delta \cdot q .
$$

We may thus have a divergence and a curl.

The method may be at once extended to space of three dimensions considered as lying in one of four ; or to one of $n$ dimensions lying in one of $n+1$, or to one of $m$ lying in one of $n$. The symbolic forms for differential parameters are then seen to be these same formulas in a different notation.

20. In his dissertation Justus Grassman claims to have established the following theorem:

Für die Wendepuncte einer allgemeinen Curve $n^{\text {ter }}$ Ordnung gilt der folgende Satz: Legt man durch $\frac{1}{2}(m-2)(m+1)$ Wendepuncte einer Curve $m^{\text {ter }}$ Ordnung eine Curve $(m-2)^{\text {ter }}$ Ordnung hindurch, so schneidet diese die erstere in $\frac{1}{2}(m-1)(m-2)$ Puncten, die gleichfalls Wendepuncte sind.

It is Dr. Slobin's intention to show that the theorem is not generally true. He applies the theorem to non-singular quintic curves having $15,11,7,3$ real inflexions, whose existence has been demonstrated by Barcroft, Klein, and others. With a proper choice of the $\frac{1}{2}(m-2)(m+1)$ inflexions as a base of a sheaf of curves of order $(m-2)$ the theorem leads to a contradiction of Plücker's formulas. We may apply the theorem to curves of higher orders, and it will be found to lead to an impossibility in many cases. The paper also takes up the classification of quintic curves as to their branches, bitangents, and multiple points.

21. This paper treats the case of two finite bodies describing elliptical orbits, and an infinitesimal body moving in the vicinity of the Lagrangian solution points. Professor Moulton discusses the properties of the orbits which are periodic in both two and three dimensions, and shows how to construct the periodic series for their cofficients. 
304 Winter meeting of the chicago section. [March,

22. The primary object of Professor Williams's paper is to prove, for scrolls of order 5, Professor Story's formula for the number of intersections of any two curves lying on the same ruled surface, i. e.,

$$
\left[C_{a, a} C_{b, \beta}\right] \equiv a \alpha+b \beta-\mu \alpha \beta,
$$

where $C_{a, \alpha}$ is a curve of order $a$ that meets each generator of the ruled surface $R_{\mu}$ of order $\mu$ in $\alpha$ points, $\alpha$ being called the grade of the curve, and $C_{b, \beta}$ is a curve of order $b$ and grade $\beta$ on $R_{\mu}$. If the curves pass through the same point of a multiple curve on the ruled surface, they are not to be regarded as intersecting there unless they lie on the same sheet. For two curves on $R_{\mu}$, of multiplicities $m$ and $n$ respectively, say $C_{a^{m_{\alpha}}}$ and $C_{b^{n} \beta}$ the formula is

$$
\left[C_{a^{m_{\alpha}}} C_{b^{n} \beta}\right] \equiv m a \beta+n b \alpha-\mu \alpha \beta .
$$

It is proved that on any scroll $R_{\mu}$ having a director line of multiplicity $\mu-1$ the number of intersections of $C_{a, a}$ and $C_{b, \beta}$ is equal to the order of the residual intersection of the two cones of orders $a$ and $b$ respectively through these curves from an arbitrary point on the multiple director as a vertex. If $S_{\nu}$ is a surface of order $\nu$, used to cut a curve $C_{b, \beta}$ from the scroll, ${ }_{2}^{5} \nu(\nu-1)+4$ points for the determination of $S_{\nu}$ can be arbitrarily chosen and $S_{\nu}$ will not break up into. $R_{5}$ and $S_{\nu-\tilde{n}^{*}} S_{\nu}$ must be chosen so that either the residual intersection shall consist entirely of generators or of generators and a curve of order less than $b$. The following values of $\nu$ were found:

$\nu=b$ and $S_{v}$ a cone for scroll I (Schwarz's classification,

$\nu=\frac{1}{5}(2 b+8+n),(n=0,1,2,3$ or 4$)$ for scrolls III, Crelle, volume 67).

$\nu=\frac{1}{5}(2 b+11+n),(n=1,2,3,4$ or 5$)$ for scrolls II, IV and $\mathrm{V}$. VI, VII, VIII, IX, X. $\nu-\frac{1}{5}(2 b+13+n),(n=0,1,2,3$ or 4$)$ for scrolls XI $\nu=\frac{1}{5}(2 b+6+n),(n=0$ or $5,1,2,3,4)$ for scrolls and XIV. XII and XIII. And for scroll $\mathrm{XV}$, for $\beta=1, \nu \geqq b-1$; for $\beta=2$,

$$
\nu \geqq 2 b-3 \text {; for } \beta \geqq 3, \nu \geqq \frac{1}{2}\left[1+(2 b+1) \beta-5 \beta^{2}\right] \text {. }
$$

